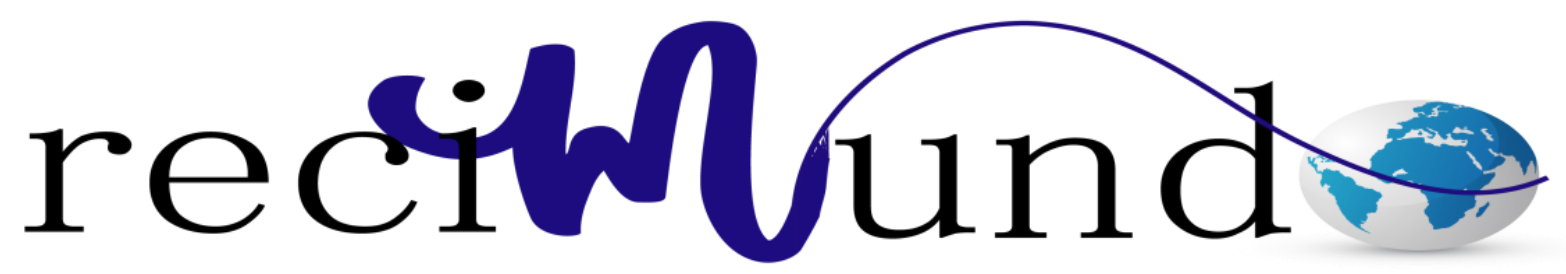

Revista Científica Mundo de la Investigación y el Conocimiento

\author{
Gladys Patricia Guevara Alban a; Alexis Eduardo Verdesoto Arguello ${ }^{\text {b; }}$ Franklin \\ Montece Mosquera ${ }^{c}$; Cristian Salomon Guevara Alban ${ }^{d}$
}

Las TIC en el proceso de la investigación científica en la educación superior

ICT in the process of scientific research in higher education

Revista Científica Mundo de la Investigación y el Conocimiento. Vol. 3 núm.3. Esp., noviembre, ISSN: 2588-073X, 2019, pp. 573-588

DOI: $10.26820 /$ recimundo/3.(3.Esp).noviembre.2019.573-588

URL: http://recimundo.com/index.php/es/article/view/623

Código UNESCO: 3205 Medicina Interna

Tipo de Investigación: Artículo de Revisión

() RECIMUNDO; Editorial Saberes del Conocimiento, 2019

Recibido: 15/09/2019 Aceptado: 23/10/2019 Publicado: 30/11/2019

Correspondencia: msgguevara@ hotmail.com

a. Diploma Superior las Nuevas Tecnologías de la Información y Comunicación y su Aplicación en la Práctica Docente Ecuatoriana; Diploma Superior en Diseño de Proyectos; Especialista en Liderazgo y Gerencia; Investigador Independiente; Guayaquil, Ecuador; msgguevara@ hotmail.com

b. Ingeniero en Sistemas e Informática; Instituto Tecnológico Superior Babahoyo; Guayaquil, Ecuador; averdesoto@itsb.edu.ec

c. Magister en Docencia y Gerencia en Educación Superior; Diploma Superior en Diseño de proyectos; Diplomado Superior en Gerencia de Sistemas; Universidad Técnica de Babahoyo; Guayaquil, Ecuador; montece@utb.edu.ec

d. Diploma Superior las Nuevas Tecnologías de la Información y Comunicación y su Aplicación en la Práctica Docente Ecuatoriana; Diploma Superior en Diseño de Proyectos; Especialista en Liderazgo y Gerencia; Instituto Tecnológico Superior Babahoyo; Guayaquil, Ecuador; cguevara@itsb.edu.ec 


\section{Las TIC en el proceso de investigación científica en la educación superior}

Vol. 3, núm. 3 Esp., (2019)

Gladys Patricia Guevara Alban; Alexis Eduardo Verdesoto Arguello; Franklin Montece

Mosquera; Cristian Salomon Guevara Alban

\section{RESUMEN}

El proceso de acumulación de saberes se estimula desde los primeros signos de vida del ser humano. La relación madre-padre e hijos deviene un cumulo de ignorancia que se busca ir resolviendo a través de distintas herramientas. Así, algo que se pudiera definir como primitivo está cargado de tanta importancia que deriva la investigación para el conocimiento, en este caso en temas como la estimulación temprana, el aprender haciendo y enseñar con el ejemplo. Es menester de esta investigación, enfrentar la realidad entre las habilidades desarrolladas, influidas y adquiridas de los profesores universitarios en cuanto al desarrollo de las Tecnologías de Información y Comunicación frente a una oleada de estudiantes universitarios absolutamente autodidactas condicionados a los contactos virtuales y alejados naturalmente del contacto físico social. La publicación digital de discusiones académicas ha aumentado. Se hace evidente el uso de espacios virtuales para la investigación. Tanto el profesor como el alumno ahora deben incluir este tipo de tecnologías para mantenerse interesados. La dinámica en aula presencial no es la misma, la mirada no va a una pizarra sino a un reproductor multimedia de la clase, es imperioso que el profesor maneje la herramienta para proyectar la seguridad que necesita el alumno de recibir la guía del instructor y que además incentive la constante búsqueda de información para generar el saber.

Palabras claves: Investigación; Universidad; TIC; Profesores; Educación 


\title{
Las TIC en el proceso de investigación científica en la educación superior
}

Vol. 3, núm. 3 Esp., (2019)

Gladys Patricia Guevara Alban; Alexis Eduardo Verdesoto Arguello; Franklin Montece Mosquera; Cristian Salomon Guevara Alban

\begin{abstract}
The process of accumulation of knowledge is stimulated from the first signs of life of the human being. The mother-father and children relationship becomes a cluster of ignorance that is sought to be resolved through different tools. Thus, something that could be defined as primitive is loaded with such importance that research is derived for knowledge, in this case on topics such as early stimulation, learning by doing and teaching by example. It is necessary for this investigation, to face the reality between the skills developed, influenced and acquired by university professors in terms of the development of Information and Communication Technologies against a wave of absolutely self-taught university students conditioned to virtual contacts and naturally away from social physical contact The digital publication of academic discussions has increased. The use of virtual spaces for research is evident. Both the teacher and the student should now include these types of technologies to stay interested. The dynamics in classroom is not the same, the look does not go to a blackboard but to a multimedia player of the class, it is imperative that the teacher handle the tool to project the safety that the student needs to receive the instructor's guide and that It also encourages the constant search for information to generate knowledge.
\end{abstract}

Keywords: Research; University; ICT; Teachers; Education. 


\section{Las TIC en el proceso de investigación científica en la educación superior}

Vol. 3, núm. 3 Esp., (2019)

Gladys Patricia Guevara Alban; Alexis Eduardo Verdesoto Arguello; Franklin Montece

Mosquera; Cristian Salomon Guevara Alban

\section{Introducción.}

El proceso de acumulación de saberes se estimula desde los primeros signos de vida del ser humano. La relación madre-padre e hijos deviene un cumulo de ignorancia que se busca ir resolviendo a través de distintas herramientas. Así, algo que se pudiera definir como primitivo está cargado de tanta importancia que deriva la investigación para el conocimiento, en este caso en temas como la estimulación temprana, el aprender haciendo y enseñar con el ejemplo.

Está demostrado que el desarrollo cognitivo del ser humano viene desde los primeros años de vida, la psicología así lo ha demostrado. Las Teorías de Piaget y de Vygostky serían las más importantes en este tema pues una nos ayuda a entender cómo interpreta el mundo un niño en distintas edades y la otra para comprender los procesos sociales que influyen en la adquisición de habilidades intelectuales. (Linarez, 2007).

Entonces, entramos en un proceso de duda que nos motiva a resolver ciertos conflictos con el papel que debemos jugar en los procesos formativos, aún más cuando por decisión personal nos hemos dedicado a enseñar.

Ana Polanco (2005) realiza un trabajo titulado "La motivación en los estudiantes universitarios", en él comienza descifrando la palabra motivación, e indica que la misma "ha sufrido un cambio progresivo, desde el punto de vista conductista, hasta las orientaciones cognitivas actuales. Su polémica gira en torno a los factores que pueden influir y la diferencia entre el interés que se presenta por una tarea. Efectivamente, es obvio que las atribuciones del éxito o fracaso, son factores que determinan la motivación de los educandos.” (Polanco, 2005). 


\section{Las TIC en el proceso de investigación científica en la educación superior}

Vol. 3, núm. 3 Esp., (2019)

Gladys Patricia Guevara Alban; Alexis Eduardo Verdesoto Arguello; Franklin Montece Mosquera; Cristian Salomon Guevara Alban

Considerando lo anterior, es deber cuestionarse de qué manera los procesos de aprendizaje van desde la inquietud personal y hasta donde podemos influir en terceros. He allí el rol del educador, y aún más complejo el educador a nivel universitario, pues recibe en aula un estudiante con una carga de aprendizaje que puede o no servir para su desarrollo profesional.

Los tiempos "modernos" muestran cambios en las maneras de conseguir objetivos, el "conocimiento práctico" o "know-how" nos obliga a valorar las habilidades naturales o adquiridas del ser y esto resulta sumamente importante cuando nos enfrentamos ahora a una sociedad virtual, a una generación completamente influida por la tecnología y que nos obliga, a los que crecimos en medio de la transición, a buscar alcanzar habilidades tecnológicas asumiendo una ignorancia cognitiva vista desde las características de Vygotsky.

La investigación es parte las tareas y responsabilidades del quehacer académico, sin embargo, se generan conflictos de distinta índole para cumplir con el requisito. Uno de ellos sería que, si el profesor universitario debe privilegiar su tarea investigativa o su labor docente, y, de otra parte, si él tiene que investigar necesariamente en la misma área de saber en la que está inscrita su docencia. En relación con la primera disyuntiva, hay quienes afirman que ese es un problema puramente formal; tal postura refleja desconocimiento de lo que es y exige la actividad de investigar, pues un investigador sabe que un proyecto de investigación riguroso implica dedicar mucho tiempo y atención, lo cual es difícilmente compatible con otras actividades, como las docentes, que también exigen una alta dedicación si se quieren realizar con la mayor calidad posible. Son múltiples las alternativas de solución de esta disyuntiva: la dedicación exclusiva y permanente a una de la dos funciones; la alternancia de periodos de investigación y de docencia; la asignación institucional de tareas investigativas, con base en el reconocimiento de méritos, o 


\section{Las TIC en el proceso de investigación científica en la educación superior}

Vol. 3, núm. 3 Esp., (2019)

Gladys Patricia Guevara Alban; Alexis Eduardo Verdesoto Arguello; Franklin Montece

Mosquera; Cristian Salomon Guevara Alban

simplemente la realización simultánea de las dos funciones, pero con la aceptación explícita de cuál de ellas será la principal, a la que la otra se subordine (Parra, 2004).

Pero también se trata de potenciar las capacidades de todos los actores, y en ello el desarrollo tecnológico tienen un papel predominante, puesto que una de las grandes ventajas no es solamente mantener la interconexión informativa, sino que con su uso los tiempos para las actividades son absolutamente eficientes y eficaces.

Es menester de esta investigación, enfrentar la realidad entre las habilidades desarrolladas, influidas y adquiridas de los profesores universitarios en cuanto al desarrollo de las Tecnologías de Información y Comunicación frente a una oleada de estudiantes universitarios absolutamente autodidactas condicionados a los contactos virtuales y alejados naturalmente del contacto físico social.

\section{Metodología.}

La metodología científica tiene como objetivo describir los pasos a seguir en la búsqueda del conocimiento. Para la realidad actual, resulta imperioso conocer cuáles son los temas de discusión en el ámbito científico para ajustar y aportar al desarrollo del aprendizaje.

El Buffet del conocimiento se encuentra en escenarios virtuales, al alcance de mayor cantidad de consumidores, es por ello que nos servimos de la herramienta virtual probada académicamente para proponer una combinación de ideas que sirva de abreboca y plato fuerte a la hora de entender como se ha visto influida la investigación académica en la educación universitaria por el desarrollo de las Tecnologías de Información. 


\section{Las TIC en el proceso de investigación científica en la educación superior}

Vol. 3, núm. 3 Esp., (2019)

Gladys Patricia Guevara Alban; Alexis Eduardo Verdesoto Arguello; Franklin Montece Mosquera; Cristian Salomon Guevara Alban

\section{Resultados.}

Hay pocas investigaciones, que apunten a la perspectiva estudiantil como fuente de datos, para identificar sus características deseables. Es claro el planteamiento en las últimas épocas, acerca de que el estudiantado es el centro del proceso de aprendizaje; pero, son escasas las ocasiones en las cuales es este grupo el que brinda su opinión acerca de sus docentes, excepto en cuanto a evaluación del desempeño en determinados cursos. En especial, a raíz de los procesos de acreditación, las instituciones educativas han otorgado al alumnado la oportunidad de evaluar, no solo los cursos recibidos, sino también a las profesoras y profesores que los impartieron. Cabe destacar que con frecuencia dichas evaluaciones enfatizan, prioritariamente, en aspectos referidos al manejo del contenido por parte de cuerpo docente, a los recursos didácticos y estrategias de aula, más que a las dinámicas interactivas, emociones y clima. En la línea señalada, la investigación desarrollada apostó a recuperar la perspectiva estudiantil acerca de las dinámicas interactivas, la caracterización del personal docente universitario y como parte de ello, el clima del aula. (Pereira, 2011).

Este tipo de investigaciones dan paso a la transformación del saber, a los cambios institucionales que deben buscarse desde las estructuras formales para aprovechar el principal recurso del conocimiento, al hombre.

En torno al control de gestión en instituciones de educación superior, este se ha caracterizado por la aplicación de controles presupuestales a razón de las exigencias gubernamentales de seguimiento de gastos en educación y a las normas de control financiero asumidas por dichas instituciones en el ámbito público. Estas exigencias comenzaron a 


\section{Las TIC en el proceso de investigación científica en la educación superior}

Vol. 3, núm. 3 Esp., (2019)

Gladys Patricia Guevara Alban; Alexis Eduardo Verdesoto Arguello; Franklin Montece

Mosquera; Cristian Salomon Guevara Alban

prevalecer rotundamente a mediados de los años 80 y principio de los 90 a raíz de la racionalización del gasto y a la crisis económica de la mayoría de los países de la América Latina. El nuevo esquema "racionalizador" del gasto obligó a los gobiernos a fortalecer los controles en la distribución y asignación de recursos al sector que finalmente obligó a las universidades a diseñar mecanismos formales e integrales de planeación institucional. El planeamiento universitario es reciente en la mayoría de las universidades nacionales en Latinoamérica, para Tûnnermann (2002) una de las características de la universidad latinoamericana tradicional, es la ausencia de una organización administrativa eficaz y el desconocimiento de la importancia de la administración académica y de la administración de la ciencia. Es común la subordinación de la investigación a las decisiones de los órganos académicos influye en la gestión de los procesos del sistema de investigación y en la asignación de recursos al sector, por lo que planificar bajo estas realidades, amerita poca autonomía en la decisiones de áreas críticas claves para el control de gestión como mecanismo válido para el mejoramiento de la dirección integral de estos sistemas. (Royero, 2003).

En los últimos veinte años, el desarrollo de las nuevas tecnologías de la información y la comunicación (TIC) ha generado cambios significativos en la sociedad. Según el "Informe Global de Tecnologías de la Información y Comunicación 2012: vivir en un mundo Hiperconectado", publicado en 2012 por el Foro Económico Mundial, vivimos en un mundo hiperconectado como resultado de la expansión de Internet, la proliferación de dispositivos móviles y accesos inalámbricos, el dominio de los medios sociales en la vida diaria y, más recientemente, el uso de la nube o cloud computing para acceder a información. Esto reconfigura la relación entre individuos, consumidores y empresas, entre ciudadanos y gobiernos; introduce 


\section{Las TIC en el proceso de investigación científica en la educación superior}

Vol. 3, núm. 3 Esp., (2019)

Gladys Patricia Guevara Alban; Alexis Eduardo Verdesoto Arguello; Franklin Montece Mosquera; Cristian Salomon Guevara Alban

nuevas oportunidades y también nuevos retos y amenazas. Los datos más recientes de Internet World Stats indican que la penetración de Internet en el mundo es del 32,7\% con un crecimiento de 528,1\% entre 2000 y 2011. Mientras que el 78,6\% de la población en Norteamérica, el 67,5\% en Australia y el $61,3 \%$ en Europa tiene acceso a Internet, los datos son significativamente menores en el resto de regiones: el 39,5\% en Latinoamérica y el Caribe, el 35,6\% en Oriente Medio, el 26,2\% en Asia y tan solo el 13,5\% en África.

Los jóvenes parecen estar desarrollando nuevas formas de comunicación, interacción, aprendizaje y búsqueda de información. Se muestran capaces de realizar tareas de forma simultánea: permanecen conectados a Internet, a través del ordenador o el móvil, al mismo tiempo que ven la televisión o atienden una clase. Constatar estos hechos ha despertado el interés de adultos, padres y profesores, que intentan comprender la brecha digital que les separa. Prensky (2001) habla de los nativos digitales, aquellos que han nacido con las TIC y se han formado con el lenguaje digital de juegos por ordenador, videos e Internet; y los distingue de los inmigrantes digitales, que han tenido que aprender a utilizar estas tecnologías, manteniendo siempre cierta conexión con el pasado. (Vidales, 2013-302).

Todo ello, exige a las instituciones de educación superior una flexibilización de sus procedimientos y de su estructura administrativa, para adaptarse a modalidades de formación alternativas más acordes con las necesidades que esta nueva sociedad presenta. Las modalidades de formación apoyadas en las TIC llevan a nuevas concepciones del proceso de enseñanza aprendizaje que acentúan la implicación activa del alumno en el proceso de aprendizaje; la atención a las destrezas emocionales e intelectuales a distintos niveles; la preparación de los jóvenes para asumir responsabilidades en un mundo en rápido y constante cambio; la flexibilidad 


\section{Las TIC en el proceso de investigación científica en la educación superior}

Vol. 3, núm. 3 Esp., (2019)

Gladys Patricia Guevara Alban; Alexis Eduardo Verdesoto Arguello; Franklin Montece

Mosquera; Cristian Salomon Guevara Alban

de los alumnos para entrar en un mundo laboral que demandará formación a lo largo de toda la vida; y las competencias necesarias para este proceso de aprendizaje continuo (Salinas, 1997). Los cambios que se dan en la institución, entre los que podemos destacar el impacto de las TIC, conducen irremediablemente a plantear un cambio de rol del profesor, de la función que desempeña en el sistema de superior. (Salinas, 2004).

Se trata de una visión de la enseñanza en la que el alumno es el centro o foco de atención y en la que el profesor juega, paradójicamente, un papel decisivo. Adoptar un enfoque de enseñanza centrado en el alumno significa atender cuidadosamente a aquellas actitudes, políticas y prácticas que pueden ampliar o disminuir la «distancia» de los alumnos distantes. La institución educativa y el profesor dejan de ser fuentes de todo conocimiento, y el profesor debe pasar a actuar como guía de los alumnos, facilitándoles el uso de los recursos y las herramientas que necesitan para explorar y elaborar nuevos conocimientos y destrezas; pasa a actuar como gestor de la pléyade de recursos de aprendizaje y a acentuar su papel de orientador. En otros trabajos (Salinas, 1997; 1998) nos hemos ocupado de los requerimientos a los profesores en este ámbito. Todo ello requiere, además de servicios de apoyo y asesoramiento al profesorado, un proceso de formación que conduzca a:

- Conocimiento y dominio del potencial de las tecnologías.

- Interacción con la comunidad educativa y social en relación con los desafíos que conlleva la sociedad del conocimiento.

- Conciencia de las necesidades formativas de la sociedad.

- Capacidad de planificar el desarrollo de su carrera profesional. (Salinas, 2004). 


\section{Las TIC en el proceso de investigación científica en la educación superior}

Vol. 3, núm. 3 Esp., (2019)

Gladys Patricia Guevara Alban; Alexis Eduardo Verdesoto Arguello; Franklin Montece Mosquera; Cristian Salomon Guevara Alban

\section{Conclusiones.}

Para Buckingham (2008:105-131) los medios digitales constituyen un aspecto indispensable de las experiencias de los jóvenes en su tiempo libre. La relación con las TIC ya no se desarrolla básicamente en el contexto escolar -como sucedía en la década de los ochenta e incluso parte de los noventa- sino sobre todo en el dominio de la cultura popular. No obstante, afirma que los cambios tecnológicos afectan a todos (incluidos los adultos) y que las consecuencias que producen las tecnologías dependen del uso que hagamos de ellas y también de otros factores externos. Por eso, para Buckingham hablar de la "generación digital" es un asunto más complejo, y señala que es preciso evitar el riesgo de sobrevalorar el papel de la tecnología en la configuración de la identidad. (Vidales, 2013-302).

Los estudiantes muestran competencias y habilidades que producen aprendizajes que no son visibles por la educación tradicional, por lo tanto, esas competencias y habilidades no son aprovechadas y percibidas por los facilitadores para la construcción de conocimiento lo que pone en tela de juicio las limitaciones de los modelos educativos vigentes para atender y aprovecharlas. Es indudable que los alcances de la tecnología y su mayor nivel de penetración en lo social va teniendo una mayor incidencia, aunque muchos de los actores desconozcan los efectos psicoepistémicos. El hecho de que surja del relevamiento un porcentaje más alto que se represente en utilizando la tecnología en el ámbito educativo, no muestra un cambio significativo dentro de las prácticas y los procesos de aprendizaje, así como tampoco de las representaciones sociales de la educación formal. El sistema educativo tradicional resulta reactivo a los cambios y se ancla a formas estáticas de tiempo, espacios, modos de transmisión, entonces el alumno no pueden representarse en él, pues el uso de la tecnología en donde muestran sus mayores destrezas 


\section{Las TIC en el proceso de investigación científica en la educación superior}

Vol. 3, núm. 3 Esp., (2019)

Gladys Patricia Guevara Alban; Alexis Eduardo Verdesoto Arguello; Franklin Montece

Mosquera; Cristian Salomon Guevara Alban

y habilidades no es entendido, valorado o aceptado en muchos casos; por ende no es aprovechado como estrategia de aprendizaje resultando un choque con la flexibilidad del lifestreaming, embodiment, realidad aumentada y ubicuidad que son herramientas que realmente despliegan las competencias digitales de las nuevas generaciones. (Fernández \& Neri, 2013).

El profesor deja de ser fuente de todo conocimiento y pasa a actuar como guía de los alumnos, facilitándoles el uso de los recursos y las herramientas que necesitan para explorar y elaborar nuevos conocimientos y destrezas; pasa a actuar como gestor de la pléyade de recursos de aprendizaje y a acentuar su papel de orientador y mediador (Salinas, 1998). Como resultado, el profesor acusará implicaciones en su preparación profesional, pues se le va a requerir, en su proceso de formación -inicial o de reciclaje-, ser usuario aventajado de recursos de información. Los profesores constituyen un elemento esencial en cualquier sistema educativo y resultan imprescindibles a la hora de iniciar cualquier cambio. Sus conocimientos y destrezas son esenciales para el buen funcionamiento de un programa; por lo tanto, deben tener recursos técnicos y didácticos que les permitan cubrir sus necesidades. En la medida en la que atendamos a los aspectos tangibles (plataforma, comunicación, materiales, funcionamiento de la red) e intangibles (comunicación pedagógica, rol del profesor, interacción, diseño de actividades, proceso de evaluación y grado de satisfacción de alumnos, profesores y gestores), seremos capaces de construir una alternativa más cercana que la educación a distancia y diferente de la enseñanza presencial. Creemos que la educación a través de la Red ofrece nuevas posibilidades de aprendizaje abierto y flexible. Pero el profesorado y el alumnado necesitan buenas condiciones de trabajo, funcionamiento adecuado de la Red, eficacia en las funciones que integran el campus virtual, calidad de los contenidos, adecuación pedagógica de las actividades, 


\section{Las TIC en el proceso de investigación científica en la educación superior}

Vol. 3, núm. 3 Esp., (2019)

Gladys Patricia Guevara Alban; Alexis Eduardo Verdesoto Arguello; Franklin Montece Mosquera; Cristian Salomon Guevara Alban

fluidez en la comunicación pedagógica, coherencia con los procesos de evaluación y acreditación. La flexibilidad debe ser una opción compatible con la rutina docente, ofreciendo seguridad de conexión y entrada al campus virtual a cualquier hora que se desee. Sólo así podemos asegurar la calidad que proporcione eficacia y satisfacción a alumnos y profesores. (Salinas, 2004).

El impacto de las TIC tanto en la universidad pública como en la privada muestra que estas herramientas fortalecen considerablemente la aprehensión de conocimiento y el enriquecimiento cognitivo; esto se constituye como el resultado de la percepción y el grado de utilización de las TIC en el proceso de enseñanza-aprendizaje. La percepción docente frente a la utilización de las TIC en los entornos de enseñanza-aprendizaje de la educación superior muestra que estas son herramientas de gran utilidad en cuanto a la construcción de una sociedad del conocimiento se trata; por tanto, la apreciación de Navarro y Alberdi (2004) sobre la posibilidad que las TIC ofrecen de acceder en forma rápida a una gran cantidad de información hace necesario que el profesor complemente su tradicional actividad transmisora de conocimientos con un esfuerzo de "aprendizaje del aprendizaje", se considera muy valedera en función del ambiente de la educación superior. Es importante considerar que la educación del siglo XXI es internacional, es decir que no tiene fronteras. (Riascos, Quinero, \& Ávila, 2009).

La comunicación, la creatividad y la gestión del cambio se han convertido en tres disciplinas que marcarán el devenir de la educación universitaria de hoy y, que deberán formar parte del universo formativo de los docentes universitarios. 


\section{Las TIC en el proceso de investigación científica en la educación superior}

Vol. 3, núm. 3 Esp., (2019)

Gladys Patricia Guevara Alban; Alexis Eduardo Verdesoto Arguello; Franklin Montece

Mosquera; Cristian Salomon Guevara Alban

La formación del profesorado en TICs conlleva no sólo ventajas, también plantea dudas e inconvenientes. Mientras que su uso y consumo además de la formación para ello permiten la “creación de entornos más flexibles para el aprendizaje, la eliminación de las barreras espaciotemporales entre docente y alumno, un incremento de las modalidades comunicativas, favorecer escenarios y entornos de carácter interactivo, el autoaprendizaje y el trabajo en grupo, salir de los muros del recinto educativo, ofrecer nuevas formas de tutoría y orientación del alumnado y favorecer la formación de carácter permanente" (Cabero, 2003: 105), la falta de una formación/preparación específica produce cierto desasosiego en los docentes, principalmente, porque estas nuevas tecnologías les ponen de relieve nuevas formas de interacción didáctica para las que no se sienten preparados, debido, generalmente, a la falta de una visión que les permita integrarlas en su metodología de trabajo. Innovación en el curriculum de las asignaturas de las diferentes carreras universitarias y una escasa o nula respuesta a las demandas sociales. En definitiva, la búsqueda de la integración de las TICs en el universo tanto formativo como no del docente pasa por un cambio, sustancial, en la concepción de las TICs y de la figura del docente. No obstante, no olvidemos que la expansión de las TIC en nuestra sociedad presenta una serie de limitaciones que Marqués (2000) resumen en: problemas técnicos y de seguridad, falta de formación y limitaciones económicas y culturales. (Marín \& Romero, 2009).

Sin embargo, se ha visto que, a lo largo de los años, sobretodo Latinoamérica va encuadrando a los nuevos tiempos. La publicación digital de discusiones académicas ha aumentado, así como el comercio de publicaciones electrónicas de distinto alcance. Se hace evidente el uso de espacios virtuales para la investigación, el uso de medios electrónico para el almacenaje de apuntes, ya casi no se escribe, se usan tablets, videograbadores o los mismos 


\section{Las TIC en el proceso de investigación científica en la educación superior}

Vol. 3, núm. 3 Esp., (2019)

Gladys Patricia Guevara Alban; Alexis Eduardo Verdesoto Arguello; Franklin Montece Mosquera; Cristian Salomon Guevara Alban

teléfonos móviles que sirven de agenta completa para interactuar. Tanto el profesor como el alumno ahora debe incluir este tipo de tecnologías para mantenerse interesados y no perderse en el mundo cibernético. La dinámica en aula presencial no es la misma, la mirada no va a una pizarra sino a un reproductor multimedia de la clase, es imperioso que el profesor maneje la herramienta, además para proyectar la seguridad que necesita el alumno de recibir la guía del instructor y en dado caso el instructor también incentivar e incluso obligar su uso.

\section{Bibliografía.}

Fernández, D., \& Neri, C. (2013). Estudiantes universitarios, TICS y aprendizaje. Anuario de Investigaciones, 10, 153-158.

Linarez, A. (2007). Desarrollo cognitivo: Las Teorías de Piaget y de Vygotsky. Barcelona: Universitad Autonoma de Barcelona.

Marín, V., \& Romero, M. (2009). La formación docenente universitaria a través de las TICS. Pixel-Bit. Revista de Medios y Educación(35), 97-103.

Parra, C. (2004). Apuntes sobre la investigación formativa. Educación y educadores, 7, 57-77.

Pereira, Z. (2011). Los diseños de método mixto en la investigación en educación: Una experiencia concreta. Revista Electrónica Educare, 15(1), 15-29.

Polanco, A. (2005). La motivación en los estudiantes universitarios. Revista Electrónica "Actualidades Investigativas en Educación", 5(2), 1-13.

Riascos, S., Quinero, D., \& Ávila, G. (2009). Las TIC en el aula: percepciones de los profesores universitarios. Educación y educadores, 12(3), 133-157.

Royero, J. (2003). Gestión de Sistemas de Investigación universitaria en América Latina. OEIRevista Iberoamericana de Educación, 33(1), 1-19.

Salinas, J. (2004). Innovación docente y uso de las TIC en la enseñanza universitaria. RUSC. Universities and Knowledge Society Journal, 1(1), 1-16.

Vidales, M. (2013-302). La relacion entre jóvenes y TICS en la investigación de la comunicación en El Salvador. Razón y Palabra, 17(1), 283. 
Las TIC en el proceso de investigación científica en la educación superior Vol. 3, núm. 3 Esp., (2019)

Gladys Patricia Guevara Alban; Alexis Eduardo Verdesoto Arguello; Franklin Montece Mosquera; Cristian Salomon Guevara Alban

$$
\text { (c) (i) (9) }
$$

\section{RECONOCIMIENTO-NOCOMERCIAL-COMPARTIRIGUAL}

CC BY-NC-SA

ESTA LICENCIA PERMITE A OTROS ENTREMEZCLAR, AJUSTAR Y CONSTRUIR A PARTIR DE SU OBRA CON FINES NO COMERCIALES, SIEMPRE Y CUANDO LE RECONOZCAN LA AUTORÍA Y SUS NUEVAS CREACIONES ESTÉN BAJO UNA LICENCIA CON LOS MISMOS TÉRMINO 